\title{
Cavitation as a method of improving the energy efficiency of oil industry enterprises
}

\author{
Denis Kuimov*, Maxim Minkin \\ Don State Technical University, Department of scientific research, Rostov-on-Don, Russia
}

\begin{abstract}
The oil industry has been and remains the cornerstone of modern energy, despite the accelerated development of green energy. This article presents theoretical studies that have confirmed the effectiveness of the effect of cavitation on changes in the properties and parameters of oil and petroleum products.
\end{abstract}

\section{Introduction}

The oil produced is generally divided into "light" and "heavy," having different both fractional composition and density and viscosity parameters. Thus, the viscosity of the produced oil is an important factor in transportation. "Light" oil due to low viscosity can be transported without preliminary preparation. The "heavy" oil must be brought to a viscosity comparable to the viscosity of the "light" oil, heating to $80-100^{\circ} \mathrm{C}$.

A deeper oil analysis indicates the complexity of the fractional composition in which organic impurities are an integral part of petroleum products. Thus, oil consists of carbon, hydrogen, sulfur, oxygen, nitrogen. In small doses, the oil may contain various metals, mainly nickel and vanadium. Hydrocarbons account for up to $75 \%$ of the total. The viscosity of "heavy" high-viscosity grades of oil when the temperature decreases reaches values at which further transportation of them becomes impossible. Including refineries can face intensive paraffination of pipelines and a corresponding decrease in throughput, which significantly complicates operation and leads to increased labor and material costs.

In connection with the current situation, modern research is aimed at creating new energy-efficient technologies and structures for influencing oil and oil products in order to improve their quality, deepen their processing and reduce viscosity. The most promising energy impact in recent years has been cavitation, both hydrodynamic and acoustic. Preliminary studies published in various sources indicate the possibility of the effect of cavitation on the fractional composition of oil and its individual components.

The effective effect of cavitation on the physical and rheological state of a liquid substance, including oil, is known and experimentally confirmed. The idea of crushing hydrocarbon chains has long been known. The first studies began back in the $60 \mathrm{~s}$ in the USSR, when a large line of hydrodynamic mixers, devices with a vortex layer, etc. appeared and were developed and launched into mass production, which were already widely used in the field of chemistry and petrochemistry, and especially in the field of

*Corresponding author: kuimov_d@list.ru 
decontamination of sewage and heavy water. Today, in the conditions of the increase in the share produced by heavy high-sulfur oil, the relevance of developing new device designs is increasing. The paper [1] presents modern knowledge and possibilities of application of cavitation technologies in the oil industry.

In this publication, an analysis of possible technologies for using cavitation was carried out, including to reduce the viscosity of crude oil, the process of ultrasonic desulfurization, as well as to increase oil recovery.

To date, the scientific literature describes many different experimental studies confirming the effectiveness of the use of cavitation technologies for changing rheological properties and affecting the fractional composition of oil, which often have controversial results. This study highlights the most effective and confirmed, in our opinion, results of the effect of cavitation on oil properties and parameters.

\section{Materials and methods}

The present work presents physicochemical effects of hydrodynamic and acoustic cavitation in terms of its effect on the structure and properties of oil and oil products. In addition, the application of energy-efficient cavitation technologies for the oil industry is discussed, including increasing the yield of "light" fractions, reducing viscosity to reduce the labor intensity of transporting high-viscosity grades of oil, and cleaning oil products from harmful impurities. Finally, we are discussing the prospects for the industrialization of energy ultrasound.

The paper presents the results of research on scientific achievements in the field of the effectiveness of the impact of various cavitation technologies on the parameters of petroleum products published over the past 30 years of study.

\section{Results of the study}

The general picture of the formation of the cavitation bubble is presented in the following form. In the vacuum phase, a gap is formed in the liquid in the form of a cavity, which is filled with saturated liquid vapor. In the compression phase, under the influence of increased pressure and surface tension forces, the cavity collapses. A significant effect of cavitation processes is associated with a high concentration of energy released during the collapse process in the treated medium. Now of collapse, the gas pressure and temperature reach significant values, and according to some data reach $100 \mathrm{MPa}$ and $\quad 1000{ }^{\circ} \mathrm{C}$, respectively [2]. The small volume of the substance explains this phenomenon when the bubble reaches its minimum radius preceding the collapse.

Based on the results of scientific research [3-5], it can be argued that the radius of the cavitation bubble at the time of collapse can reach, as a rule, $10^{-7}-10^{-8} \mathrm{~m}$, against the radius in an equilibrium state of $10 \cdot 10^{-6} \mathrm{~m}$. It is known that the cavitation "germ" receives its maximum radius Rmax in the rarefaction phase (acoustic wave, the movement of the ferromagnetic element along the liquid flow). The Rmax value is significantly greater than the minimum radius Rmin preceding the collapse process. As a result, the equation of energy stored in the cavitation bubble can be written as follows:

$$
W=\frac{4}{3} \pi R_{\max }^{2} P_{0}
$$

where, $\mathrm{P}_{0}$ - is the pressure of the surrounding liquid, $\mathrm{Pa}$; W - energy, $\mathrm{J}$.

If the following values $\mathrm{R}_{\max }=100 \mu \mathrm{m}, \mathrm{P}_{0}=0.1 \mathrm{MPa}$ are taken as the initial data, then the amount of energy stored by the cavitation bubble will be $4 \cdot 10^{-7} \mathrm{~J}$, which, when such a bubble is compressed by 1000 times, will allow the energy density to be $10^{15} \mathrm{~J} / \mathrm{m}^{3}[5]$. 
The energy generated from the collapse of cavitation bubbles is enough to overcome the energy of compounds and the destruction of molecules. Let's consider possible processes of oil treatment with cavitation effect.

Oil is a high molecular weight, heterogeneous liquid whose molecules are difficult to orient at atmospheric pressure and normal temperature. When external pressure is applied to oil in several hundred atmospheres, the molecules polarize, counteracting external forces and maintaining the equilibrium of the system. If the external pressure is abruptly removed, then the internal forces will begin to break the macromolecules into smaller components, moreover, the density of the product decreases. This principle is the basis of oil treatment in order to change its structure.

Table 1. Bond breaking energy of some types of connections.

\begin{tabular}{|c|c|c|}
\hline Type of link & $\begin{array}{c}\text { Type of } \\
\text { connection }\end{array}$ & $\begin{array}{c}\text { Bond break } \\
\text { energy, } \\
\mathbf{k J} / \mathbf{m o l}\end{array}$ \\
\hline C-COOH & Acids & 230 \\
\hline C-C & Paraffin & 332 \\
\hline C-N & Amines & 334 \\
\hline C-H & Paraffin & 413 \\
\hline C $=\mathrm{C}$ & Olefins & 588 \\
\hline
\end{tabular}

\subsection{Reduced viscosity of crude oil by cavitation}

In recent years, the world has seen a trend of depletion of so-called "light" oil fields and an increase in the share of "heavy" oil. The use of "heavy" oil brings a lot of difficulties to the oil industry due to the significant energy costs necessary for the transportation of viscous "heavy" oil.

Unlike "light" grades, "heavy" grades of oil have a large share of asphaltenes, heavy metals, sulfur. At the same time, asphaltenes, being complex molecules, are insoluble in straight chain hydrocarbons. Therefore, during transportation, it is possible to deposit asphaltenes causing high-pressure drops in the pipelines, thereby complicating transportation. Heavy oil viscosity exponentially increases as asphaltenes content increases. Therefore, preventing serious agglomeration of asphaltenes is key to reducing the viscosity of crude oil, especially for heavy and superheavy oil.

In some cases, viscosity reduction is achieved by either diluting the "heavy" oil with a "lighter" one, heating the pipelines, and emulsifying the "heavy" oil with water. However, all methods presented greatly increase-operating costs.

A number of works have proved that the use of cavitation can reduce the viscosity of crude oil. In operation [6] it is shown that ultrasonic cavitation oil treatment is capable of reducing viscosity of treated crude oil by $15 \%$. And in operation [7], during cavitation treatment, not only a $57 \%$ reduction in viscosity was achieved, but also a change in the fractional composition of the oil product was established.

Despite a large number of theoretical and experimental studies aimed at studying and confirming the effect of reducing viscosity-using cavitation, it is still not completely clear by what processes the viscosity decreases, but there are a number of theories. Most often, the decrease in viscosity is explained by the effect of cavitation on asphaltenes. 
According to [8], the asphaltenes are in the form of micellar nuclei and are stabilized by adsorbed resins. In this case, the ratio of resins to asphaltenes are key parameters that affect the viscosity of oil.

In work [9] positive influence of acoustic cavitation on asphaltenes of crude oil is confirmed. According to experimental studies in this field, the effect of acoustic cavitation leads to the decay of asphaltenes. The interaction between the asphaltene particles decreases with a decrease in their size, which subsequently leads to a decrease in viscosity. Thus, according to the conclusions made in work [10], cavitation efficiency is due to both local temperature increase in the area of cavitation bubbles collapse and the effect of cavitation directly on asphaltenes.

Paraffins along with asphaltenes give colloidal properties to petroleum products. The presence of paraffin series hydrocarbons (alkanes) in oil determines the structure and properties of the dispersion system. Oil does not have viscosity subject to the laws of Newton, Poiseuil, Stokes, since long randomly located paraffin and resin molecules form some flexible lattice in which the solution is located. Therefore, the system exerts considerable resistance to shear forces. Cavitation breaks a continuous chain, destroying the bonds between individual parts of molecules. Van der Waals ties break. The acceleration of paraffin dissolution is due to the intensification of oil mixing at the oil-paraffin border and the action of pressure pulses that, as it were, spray paraffin particles.

There are a number of studies according to which the long-term effect of cavitation on oil leads to disruption of bonds in paraffin molecules. As described above, the bond energy of the various hydrocarbons varies over a fairly wide range. Accordingly, when hydrodynamic cavitation affects the oil product, the weakest bonds of molecules begin to decay in it. The weakest bonds are bonds in the middle of the molecule, where it is easier to tear off part of the molecule than from the end, which accordingly leads to a peculiar fragmentation of long hydrocarbon chains into smaller ones.

Based on the above, any compound in the feedstock to be treated is capable of destruction when the energy is supplied thereto is sufficient to overcome the corresponding bonding energy.

Under normal conditions, the medium and long chain alkanes are in the dispersed phase. According to the results presented in work [11], the effect of a single pulse of hydrodynamic cavitation on medium-chain alkanes $\mathrm{C}_{21}-\mathrm{C}_{38} 4.9 \%$ appeared in the paraffin composition. Hydrocarbons $\mathrm{C}_{6}-\mathrm{C}_{20}$. These products according to the conclusions [11] were formed due to the decomposition of alkanes $\mathrm{C}_{25}-\mathrm{C}_{35}$.

The results obtained in the work [12] confirmed the character of the main directions of conversion of short-chain alkanes under the influence of hydrodynamic cavitation. The presence of such hydrocarbons in the composition of oil and gas condensate allows, due to hydrodynamic cavitation, to slightly increase the content of alkanes S18-S22 (boiling limits of $310-370^{\circ} \mathrm{C}$ ) and, to a greater extent, $\mathrm{C}_{8}-\mathrm{C}_{13}$ (boiling in the range of $125-235^{\circ} \mathrm{C}$ ).

\subsection{Increased yield of "light" fractions}

The possibility of implementing the cavitation process is largely determined by the parameters of the treated medium, including:

- temperature;

- number of gas inclusions;

- presence of insoluble impurities.

The use of crude crude oil as a raw material for the implementation of the processing process makes it possible to fulfill all these conditions and obtain the maximum possible concentration of cavitation bubbles in the range from $10^{11}$ to $10^{12} \mathrm{~m}^{-3}$, which is confirmed by the results of scientific research given in the works [4]. These properties of crude oil 
make it possible to conclude that the use of cavitation in oil treatment technologies is highly promising by hydrodynamic and acoustic cavitation.

It is known that the collapse of cavitation cavities leads to a local increase in temperature and pressure, initiating a kind of microcracking process.

In general, there are two types of cracking used in the oil industry, divided into thermal and catalytic. Thermal, based on the name, involves the use of high temperatures to break the bonds of molecules.

The authors in scientific work [13] presented a study confirming that cavitation treatment can not only reduce the amount of paraffins in the processed product, but also increase the proportion of resinous and asphaltene hydrocarbons.

In work [14], experimental studies have found that the cavitation effect on oil can initiate partial removal of dissolved gases and light boiling hydrocarbons from the liquid phase. At the expense of it and reactions of consolidation concentration of components of oil, the boiling-away above $200{ }^{\circ} \mathrm{C}$ increased. The subsequent distillation of the processed product which is followed termolizy showed increase in an exit of the fractions boiling up to $300{ }^{\circ} \mathrm{C}$. Thus, by authors of work [14] it is established that the number of fractions with a temperature of boiling of $120-200{ }^{\circ} \mathrm{C}$ increased by $17 \%$ and also increase of concentration of paraffin hydrocarbons, naphthenes and aromatic C8 connections - C10 is noted. The number of fractions with a boiling point in the range of $28-350$ increased by $4-7 \%$. However, the authors of the work [8] also found that the absence of an effect on the product treated with cavitation for a long time leads to its initial state.

\subsection{Desulfurization of crude oil}

In addition to the difficulty of reducing viscosity, the process of primary purification of crude oil from harmful impurities that negatively affect the equipment presents significant difficulties. The main impurities that negatively affect the active properties of the catalysts include metals, nitrogen-containing compounds (aniline, pyridine, quinoline). At the same time, sulfur, not being a harmful impurity in cracking, contributes to the process of coke formation, which requires a reduction in sulfur-containing impurities in the primary treatment.

Among the main methods of oil desulphurization, the most common and tested technologies are:

- hydrotreating;

- adsorption methods;

- alkaline cleaning;

- Merox.

All these methods have proven effectiveness in oil desulphurization processes, and have already found their application in many refineries of various capacities. However, their application is difficult not only for mini-refineries. Firstly, the disadvantage common to all methods is the impossibility of complex purification of oil from all sulfur compounds, and their in oil products can contain up to 250 types. Secondly, almost all desulfurization processes are not designed to treat "heavy" fractions of oil, which, given the annual increase in the share of "heavy," dirty grades in the total volume of oil produced, leads to obsolescence and the need to change technologies and the general modernization of oil refining.

Thus, the main negative processes of applying traditional methods of desulfurization of oil and oil residues can be distinguished:

1. high energy intensity;

2. selectivity of methods;

3. irretrievable losses of expensive catalysts; 
4. complexity of the system.

In crude oil, sulfur is divided in its properties into active and inactive sulfur. Active sulfur includes sulfur compounds that can react directly with metals. The active sulfur includes elemental sulfur itself, hydrogen sulfide and mercaptans. Inactive sulfur (passive), respectively, includes compounds that do not react with metals and include sulfides, disulfides, thiophene and thiophane.

The processes used in oil refineries are primarily aimed at removing active sulfur, which can be harmful during operation, storage or transportation. Inactive sulfur, despite the absence of possible direct harm during operation, has low thermal stability, which leads to its decomposition to hydrogen sulfide when heated.

Among the existing methods of oil desulfurization, environmentally friendly methods are being studied most actively in recent years, among which cavitation energy effects, both acoustic and hydrodynamic, are distinguished. The method of sonocatalytic desulfurization of oil products is tested in work [15]. According to this study, the use of ultrasonic cavitation can increase the degree of desulfurization up to 4 times.

Over the past few decades, many works have been carried out to study and develop alternative methods for desulfurization of both crude oil and its products, biodesulfurization methods, ion catalysis, oxidative desulfurization [16-24] have been developed. The analysis and conclusions presented in this paper do not fully disclose the ability of cavitation to implement the oil desulfurization process.

In operation [25] hydrothermal diesel fuel is used as the treatment product. The treatment was carried out in an ultrasonic apparatus with an acoustic wave frequency of 28 $\mathrm{kHz}$ in the presence of reagents: hydrogen peroxide (30\%), acetic acid $(99 \%)$ dimethylphomamide (DMF 96\%). The results presented in the work do not allow us to conclude that the method is promising, primarily due to insufficient desulfurization, since the method is suitable only for desulfurization of low-sulfur diesel fuels. And the use of additional reagents in the processing process further reduces interest in the technology.

The study presented in the paper [26] is more interesting. It analyzed the treatment of "heavy" grades of oil using hydrodynamic cavitation. The paper presents the procedure of heavy fuel oil treatment by hydrodynamic cavitation at temperature of treated material $800 \mathrm{~S}$, atmospheric pressure for 10-15 minutes.

Despite the fact that the treatment was mainly aimed at reducing the viscosity of the treated oil product, analysis of the parameters of the initial oil product and the treated oil, it is possible to conclude that the sulfur content decreases from 3.35 to 2.87. The decrease in sulfur content in heavy oil after cavitation treatment is explained by different values of the bonding energy. The C-S bond energy is lower than the C-C bond energy $(276 \mathrm{~kJ} / \mathrm{mol} \mathrm{vs}$. $334 \mathrm{~kJ} / \mathrm{mol}$ ), resulting in the breakdown of primarily C-S bonds. The positive results presented in the publication, including a decrease in the density of the treated material and a decrease in the sulfur content, make it possible to conclude that the method of hydrodynamic oil treatment is promising. However, in order to increase the effect of reducing the sulphur content, studies aimed at desulfurizing oil should be carried out.

In the works [27-30], the first steps were taken in the development of the desulfurization of oil by hydrodynamic cavitation. In the presented works, the possibility of rupture of sulfur compounds by intense exposure to cavitation is shown. As a result of the treatment, the sulfurous compound was precipitated as micelles. Despite the presented possibility of oil desulfurization by hydrodynamic cavitation, these works miss the study of the effect of cavitation on the accompanying hydrocarbon chains, various impurities, and in general the fractional composition of the processed product and treated product is not evaluated. 


\section{References}

In recent years, the key problems of the oil industry have become the general weighting of oil, depletion of light grades, and an increase in the amount of harmful impurities. Cavitation technologies, as developing energy-efficient green technologies, have great potential for application both in the field of deepening the processing of extracted oil products and in the stages of cleaning oil from harmful impurities.

Low energy costs for the creation of both hydrodynamic and acoustic cavitation, combined with high efficiency, will remain in the near future the most promising and relevant development. Currently, a number of structures of flow-type hydrodynamic cavitation devices have already been created for the implementation of oil treatment processes on an industrial scale, but the effectiveness of many structures has yet to be proved in the future. At the moment, it can be confidently argued that under certain circumstances, the energy generated by the collapse of cavitation bubbles is sufficient to break a number of hydrocarbon compounds. To increase the efficiency of a particular technology, it is necessary to conduct experimental studies on the possibility of breaking the bonds of certain types of compounds, which in the future will help to create technologies for the selective effect of acoustic and hydrodynamic cavitation on the fractional composition of oil. The technologies currently being developed should focus on the treatment of high viscosity and heavy grades of oil with a high content of asphaltenes, since a higher initial viscosity of crude oil leads to higher cavitation efficiency.

Here are some examples:

1. Balasubrahmanyam Avvaru, Natarajan Venkateswaran, Parasuveera Uppara, Suresh B. Iyengar, Sanjeev S. Katti, Ultrasonics Sonochemistry, 42, 493-507 (2018), ISSN 13504177.

2. Ivanitsky G.K. Collection of scientific articles Modern Science, 2 (7) 52-58.

3. K.Yu. Yakimenko, A.A. Wengerov, A.E. Brand Basic research. 5(3) 531-536 (2016)

4. Nurullaev V.Kh. Transportation and storage of oil products, 1. 38-42 (2017)

5. Koryagin V.A. Burning water-fuel emulsions and reducing harmful emissions. C-P, 1995.

6. A. Chakma, F. Berruti, Ultrasonic visbreaking of Athabasca bitumen, in:Proceedings of the 5th UNITAR International Conference on Heavy Crude and TarSands, 1991

7. C. Shi, W.Y. Yang, J. Chen, X. Sun, M. Pei, Application and mechanism of ultra-sonic static mixer in heavy oil viscosity reduction, Ultrason. Sonochem. 37 648-653 (2017)

8. U.K. Gollapudi, S.S. Bang, M.R. Islam, Ultrasonic treatment for removal of asphaltene deposits during petroleum production, in: SPE 27377, SPE Intl.Symposium on Formation Damage Control, Lafayette, Louisiana, U.S.A., 1994

9. I. Najafi, M. Amani, Asphaltene flocculation inhibition with ultrasonic wave ra-diation: a detailed experimental study of the governing mechanisms, Adv. Petrol.Explor. Dev. 2 (2) 32-36 (2011)

10. Salehzadeh, A. Akherati, F. Ameli, B. Dabir, Experimental study of ultrasonicradiation on growth kinetics of asphaltene aggregation and deposition, Can. J.Chem. Eng. 94

(11) 2202-2209 (2016)

11. Torkhovsky V.N., Vorobyev S.I., Egorova E.V., Antonyuk S.N., Gorodsky S.N. Vestnik MITHT (Fine Chemical Technologies). 9 (4). 59-69. (2014).

12. V.N. Torkhovsky, S.N. Antonyuk, S.I. Vorobyov, M.V. Nikolaev Fine Chemical Technologies 12 (5) (2017) 
13. V.K. Nurullaev Transportation and storage of oil products. 1 (2017)

14. V. N. Torkhovsky, E. V. Chizhevskaya, S. N. Antonyuk [et al. ] // On the use of simulated distillation in studying the effect of hydrodynamic cavitation on the conversion of hydrocarbon raw materials/Unconventional natural resources, innovative technologies and products: Collection of scientific works/Under the general editorship of V. Zelenkov.. - Moscow: Russian Academy of Natural Sciences, 2016. - Page 8697.

15. Grideva E.S. Desulfurization of petroleum products under the influence of ultrasound: specialty 05.17.08 "Processes and devices of chemical technologies": dissertation for the degree of candidate of technical sciences/Grideva Ekaterina Sergeevna. - Moscow, 2010. - 136 pages.

16. H. Chen, H.W.J. Zhang, J.M. Chen, Y.B. Cai, W. Li Technol., 99 6928-6933 (2008)

17. F. Li, Z. Zhang, J. Feng, X. Cai, X. Ping Biotechnol., 127, 222-228. (2007)

18. K. Tawara, T. Nishimura, H. Iwanami, T. Nishimoto, T. Hasuike Ind. Eng. Chem. Res., 40 2367-2370 (2001)

19. L. Huang, G. Wang, Z. Qin, M. Dong, M. Du, H. Ge, X. Li, Y. Zhao, J. Zhang, T. Hu, J. Wang Environ, 106, 26-38, (2011)

20. Y. Mochizuki, K. Sugawara Removal of organic sulfur from hydrocarbon resources using ionic liquids Energy Fuels, 22, 3303-3307, (2008)

21. L.N. He, H.M. Li, W.S. Zhu, J.X. Guo, X. Jiang, J.D. Lu, Y.S. Yan Ind. Eng. Chem. Res., 47, 6890-6895, (2008)

22. R. Schmidt [bmim] AlCl4 ionic liquid for deep desulfurization of real fuels Energy Fuels, 22, 1774-1778, (2008)

23. N.S. El-Gendy, J.G. Speight Handbook of Refinery Desulfurization, CRC Press Taylor and Francies group, 213-233, (2015)

24. F.T. Li, R.H. Liu, J.H. Wen, D.S. Zhao, Z.M. Sun, Y. Liu Desulfurization of dibenzothiophene by chemical oxidation and solvent extraction with Me3NCH2C6H5Cl-2ZnCl2 ionic liquid Green Chem., 11 (2009), pp. 883-888.

25. Yongchuan Dai, Yutai Qi, Dezhi Zhao, Huicheng Zhang, An oxidative desulfurization method using ultrasound/Fenton's reagent for obtaining low and/or ultra-low sulfur diesel fuel, Fuel Processing Technology, 89 (10), 927-932 (2008), ISSN 0378-3820.

26. Mohammad Askarian, Ali Vatani, Mohsen Edalat, Journal of Petroleum Science and Engineering, 151, 55-61 (2017), ISSN 0920-4105.

27. Kuimov, D.N., Minkin, M.S., Lukyanov, A.D. Materials Science Forum, 870, 671-676. (2016)

28. Kuimov, D., Minkin, M. MATEC Web of Conferences, 132, 03016 (2017)

29. Kuimov, D., Minkin, M. Serbian Journal of Electrical Engineering, 14 (3), 323-331 (2017)

30. Kuimov, D., Pavlenko, A., Minkin, M. MATEC Web of Conferences, 226, 02028 (2018) 